\title{
Promoting Attachment and Emotional Regulation of Children with Complex Trauma Disorder
}

\author{
Dianna Aideuis
}

\begin{abstract}
Complex trauma is the result of repeated or chronic traumatic experiences in childhood. The traumatic experiences have a pervasive impact on the child's physical, sensory, emotional, cognitive and social growth. This paper discusses how the proposed diagnostic category, Complex Trauma Disorder, identifies clusters of symptoms and behaviors that suggest the need for a multimodal approach to therapy. Application of each treatment modality is described with the emphasis on resolving the symptoms of complex trauma and development of a secure attachment. Keywords: complex trauma, attachment, emotional regulation, Dyadic Developmental Psychotherapy, Traumafocused Cognitive Behavioral Therapy, Eye Movement Desensitization and Reprocessing.
\end{abstract}

\section{Introduction}

Attachment has been studied for many years. Bowlby (1988), Ainsworth (1978) and others have documented their observations and research concerning attachment. Through the work of these early pioneers we are able to recognize the factors that promote secure attachments such as experiences through facial expressions and eye contact, emotional attunement and play. A secure attachment provides a protective source of mutual regulation. This is accomplished through mirroring the child's responses including facial expressions, tone and excitement or calm and the child gradually develops the ability to self-regulate (Davies, 2004). The child becomes securely attached when the parent/caregiver is available and able to meet the child's needs in a responsive and appropriate manner. Secure attachment provides a sense of safety, and assistance with regulation of affect and arousal. The parent/caregiver's ability to read the infant's affect accurately and provide stimulation or soothing helps the infant to self regulate and form a secure attachment with the caregiver. Secure attachment promotes the expression of feelings, through parent/caregiver modeling and teaching the child how to cope with problems that arise (Hughes, 2007). The child will freely explore with the parent/caregiver present and will interact with strangers. The child will also be visibly upset when the parent/caregiver leaves and will be happy upon their return. The child who develops a secure attachment has more confidence in exploring the environment and is ready to learn new things (Davis, 2004).

In contrast, when the caregiver is neglecting or abusive to the child, the child's ability to selfregulate and form a secure attachment is limited. Neglect and abuse significantly effect attachment and brain development. Prolonged, severe or unpredictable stress, including neglect and physical, emotional, and sexual abuse, as well as domestic violence during early childhood, can cause the brain's development to be altered (Solomon \& Siegel,2003). The child's experiences have a negative impact on his/her physical, sensory, emotional, cognitive, and social growth. Neglect alone can cause neuronal pathways to wither and die, and as a result, the child may not achieve the usual developmental milestones. Children who have been raised in abusive, neglectful environments, where their parents totally disregard their children's need for comfort, stimulation and affection may be at risk for developing Reactive Attachment Disorder (RAD) (DSM-IV, 1994) (U.S. Department of Health and Human Services, Oct 2001).

RAD (DSM-IV, 1994) is a psychophysiologic condition with markedly disturbed and developmentally inappropriate social relatedness in most contexts that begins before age five years and is associated with grossly pathological care. This pathological caregiving behavior may consist of any form of neglect, abuse, mistreatment or abandonment. However, the diagnosis of RAD does not adequately 
describe the physiological and psychological problems traumatized children present; also trauma and traumatic grief are not age limited.

Many children experience stressful events and are sometimes faced with painful situations, such as death of a close elderly relative or parent's divorce, but these events would not usually be considered traumatic (Cohen, Mannarino \& Deblinger, 2006). Traumatic events are characterized as sudden unexpected events, shocking in nature or death/threat to life or body integrity as well as subjective feelings of intense horror, terror or helplessness (American Psychiatric Association, 2000). Prolonged, severe or unpredictable stress, including physical, sexual, emotional abuse and neglect are considered traumatic events.

Post-traumatic stress disorder (PTSD) (DSM-IV, 1994) is a term used to explain consequences of exposure to, or confrontation with stressful events that the person experiences as highly traumatic. The experience must involve actual or threatened death, serious physical injury, or a threat to physical and/or psychological integrity. The diagnosis of PTSD fails to adequately describe the effects that trauma has on children. It fails to recognize the child's loss of a sense of safety, trust, and self-worth. The child experiences a sudden separation or loss of their attachment figure, and withdraws or shows disinterest in social interactions (e.g., child refuses to accept comfort from others even in times of acute distress) (Osofsky,2004). There is also a tendency for the child to be revictimized. Children who are physically or sexually abused are more likely to be abused as adults (van der Kolk, 1996).

In recent years, leaders in the treatment of child trauma research have introduced a new diagnosis that more adequately describes the effects of trauma and traumatic grief in children. Complex trauma is being considered for inclusion in the next revision of the DSM (van der Kolk, 2005). Complex trauma is used to address the multifaceted nature of trauma experienced by children when violence, neglect, and fear form the fabric of their early existence. This proposed diagnosis is being used to better define the pervasive experiences of children who have been abused and or neglected at any age. Complex trauma is characterized by chronic difficulties in many areas of emotional and interpersonal functioning. There are seven domains which describe the complexity of symptoms and behavioral characteristics that make up this disorder. The first domain of complex trauma is that of attachment: the child experiences uncertainty about the reliability and predictability of the world; social isolation; distrust and suspicion; interpersonal difficulties such as conflict with parents/caregivers, siblings, peers, and teachers; difficulty attuning to the emotional state of others; and misunderstanding and misinterpretation social cues. The second domain is biological: the child experiences symptoms such as sensory processing issues manifested as hypersensitivity to physical contact; analgesia (absence of sensitivity to pain); upper body weakness; sensitivity to sounds, tastes and smells; various somatic illnesses manifested as headaches, stomachaches, and limb pain; and increased medical problems. The third domain includes affect or emotional regulation: difficulty de-escalating; chronic and pervasive depressed mood or sense of emptiness; chronic preoccupation with suicide; difficulty describing feelings and internal experience; and explosive anger or inhibited anger. The fourth domain is dissociation: distinct alterations in states of consciousness, amnesia, depersonalization and de-realization. The fifth domain is behavioral control: poor modulation of impulses, self-destructive behaviors (self injury), aggressive behavior, sleep disturbances, eating disorders, substance abuse, and oppositional behaviors or excessive compliance. The sixth domain is cognition: difficulty in attention regulation and executive functioning, problems focusing on and completing tasks, difficulty planning and antic ipating events, learning difficulties, and problems with language development. The seventh domain is self-concept: lack of continuous and predictable sense of self, low self-esteem, feelings of shame and guilt, generalized sense of being ineffective in dealing with the environment and belief that one has been permanently damaged by the trauma (van der Kolk, 2005). These symptoms are likely to interfere with developmental tasks which create a more complex clinical picture as the child matures (Moran, 2007). 
Due to the complexity of the symptoms and the significant impact trauma has on the life of the child, multiple evidence-based practice therapy models may be necessary. Working with children with complex trauma requires a dynamic process which is fluid and completely individualized for each of the children and their families. It is essential that the therapist have knowledge and understanding of a variety of treatment models, so they can thoughtfully apply them (Amaya-Jackson \& DeRosa, 2007). Therefore it is criticalfor the therapist to have a "tool bag" of strategies/therapeutic models that are evidence-based from which to choose.

During the assessment phase, information is gathered regarding the child's behavioral issues, and emotional symptoms, and any history of trauma/traumatic grief, abuse and/or neglect. If the child has been adopted or is in foster care, a complete history is requested as to this child's earlier life experiences and attachment history. The present parent's/caregiver's own attachment history is also explored. This historical assessment of the child and parents/caregivers is done outside the presence of the child in order to minimize affect dysregulation (an emotional response that is not well modulated by the child) and decrease the child's reactivity to possible triggers (trauma reminders).

If it appears that the child has symptoms consistent with sensory processing disorder the child is referred to Occupational Therapy for further evaluation and treatment. Movement, music, bodyawareness exercises, self-calming techniques, and sensorimotor play provide the most effective therapy for children who experience extreme physical vulnerability and/or who have distorted body concepts (James, 1989).

The first meeting after the initial assessment is with the parents/caregivers to provide psychoeducation regarding the effects of maltreatment and trauma on the brain (National Clearinghouse on Child Abuse and Neglect Information, 2001). They are also provided information on treatment models and behavior management. They are provided articles and websites to obtain additional information. The parents/caregivers are encouraged to read Off Road Parenting (Pacifici, Chamberlain \& White 2002) in order to provide them with a guide for behavior management. A parent - professional partnership model is utilized to help parents/caregivers to be better able to care for their child. A need for support to families rearing children with serious emotional and mental health challenges has sparked a national family movement (McCammon, Spencer \& Friesen, 2001). It is crucial that the therapist create an alliance with the parents/ caregivers and assist them in developing an intersubjective (reflective, nonverbal attunement/awareness and interaction between the parent/caregiver and the child) dialogue with the child. The therapist and parent/caregiver are attuned (responsive) to the child's subjective experience and reflect this back to the child (e.g. the child perceives the parent's/caregiver as mean and uncaring when the child is restricted from an activity or event as a consequence to misbehavior: the parents/caregiver provide empathy (through verbal and non-verbal cues) and discuss the child's feelings and provide understanding). This is a key component in the Dyadic Developmental Psychotherapy (DDP) treatment model (Hughes, 2006). Intersubjectivity provides a means in which the child can feel safe. When a safe environment is created at home and in the therapist's office the child is then able to express their inner most thoughts and feelings as they begin the process of developing a coherent narrative about their experiences. The intersubjective experience provided in therapy enables past conflicts, misunderstandings, separations, traumas and misattunement (mistakes on the part of the parent/caregiver) to become integrated into the family narratives/story where clarity and understanding is experienced by the child. The child is then able to develop a new deeper meaning of their own experience (Hughes, 2007). When the child and family are engaged in this process, it often leads to the development of a more coherent narrative for the child. The goal is to resolve shame and cognitive distortions, and promote a feeling of safety in which the child can reach their developmental milestones, and develop pro-social behaviors and closer relationships with parents/caregivers. 
Dyadic Developmental Psychotherapy (Hughes, 2007) is an evidence based practice model. This model takes a collaborative approach with the parents/caregivers. Throughout treatment, the parents are asked to take their cue from the therapist as they learn the art of intersubjective dialogue as the central activity of treatment (Hughes, 2007). The child's inner most thoughts and feelings are then explored utilizing this model. Positive attributes, successes, and behaviors are discussed. Misbehaviors are explored and the parents/caregivers are encouraged to discover what might be behind the misbehaviors utilizing intersubjective dialogue (e.g. the child might steal food and hid it in their room, the parents/caregivers need to approach the child in an accepting manner, their tone needs to be calm and caring, they need to be curious about the incident and explore what meaning the child gives to hiding the food and then further discuss how the issue can be resolved). The parents/caregivers are also encouraged to help the child repair or make amends for the misbehavior. The parents/caregivers are also encouraged to make amends when they make mistakes with their child. The parents/caregivers are taught the PACE stance (Hughes, 2007). The PACE stance is Playful, Accepting, Curious, and Empathetic (Hughes, 2007). This therapy model requires several hours of training at master's degree or higher.

Playfulness is done whenever the child may need some help with accepting a mistake they made or to help improve their mood. Playfulness might be used when a child has trouble waking in the mornings. For example the parent might sing a song to the child or mirror the child being sleepy and be yawning and stretching and making silly faces as if they are waking up themselves.

Acceptance of the child is extremely important. As in the example above, if the child always has trouble waking up (assuming that there are no medical causes for this dilemma) the parent accepts this about their child and adjustments are made to help the child in a playful, loving way.

It is also important for parents/caregivers to use curiosity whenever there is a problem or concern for the child's behaviors. The parent/caregiver might talk with the child and ask questions about what might make it easier for the child to wake up and get dressed each morning. The parent would utilize the intersubjective dialogue as a means of communication between the parent/caregiver and child. The intersubjective dialogue is filled with non-verbal cues as well as words that express acceptance, curiosity, and empathy. The exchange provides an opportunity for the parents/caregiver to co-regulate the child and provide an opportunity for a reciprocal relationship in which to gain greater understanding of each other.

DDP is utilized throughout the course of treatment. The parents/caregivers are also encouraged to continue using PACE and the intersubjective dialogue with their child at home.

At the next appointment identification of feelings and coping skills are taught. The Coping Skills Game (Childswork/ Childsplay, LLC. 1996) is played with the parents/caregivers and the child. The family is given a list of coping skills to take with them in order to practice at home. The child is also introduced to a biofeedback game, Journey to the Wild Divine (Bell, 2003). This game helps the child to understand how to control their emotions, using breathing techniques and muscle relaxation in which to reduce stress and provide a sense of balance. At this point another evidence-based practice therapy model, Trauma Focused Cognitive Behavioral Therapy (TF-CBT) is being utilized. This model stresses the importance of feelings identification, development of coping skills or affect regulation by teaching deep breathing and muscle relaxation to help regulate emotions and behavior. Psycho-education which provides information for parents/caregivers and children on domestic violence, and physical, sexual, and emotional abuse is another key component of the TF-CBT model. Also, in this model, development of the coherent trauma narrative is done through gradual exposure and cognitive processing to reduce the physical and emotional impact and avoidance of trauma reminders (Cohen, Mannarino \& Deblinger, 2006). This therapy model requires several hours of advanced training at master's degree level or higher. 
During the next appointment coping skills are reviewed and the child and parent/caregiver are asked if they used any coping skills in the past week and which one(s) they used. The child is also given an opportunity to continue playing the Journey to the Wild Divine Game while the therapist is meeting with the parents. Then the cognitive triangle is introduced (Cohen, Mannarino \& Deblinger, 2006), first to the parents then to the child. The cognitive triangle is used to explore negative thoughts, feelings and behaviors the child may be feeling. The easiest part of the triangle to change is our thoughts. Positive and negative thoughts are explored with the child. The feelings associated with the thoughts are identified and then positive and negative behaviors are explored. The goal is to help the child develop appropriate positive thoughts to substitute for the negative thoughts. When the negative thought is changed, the negative feeling is also changed and this in turn changes the negative behavior. The cognitive triangle:

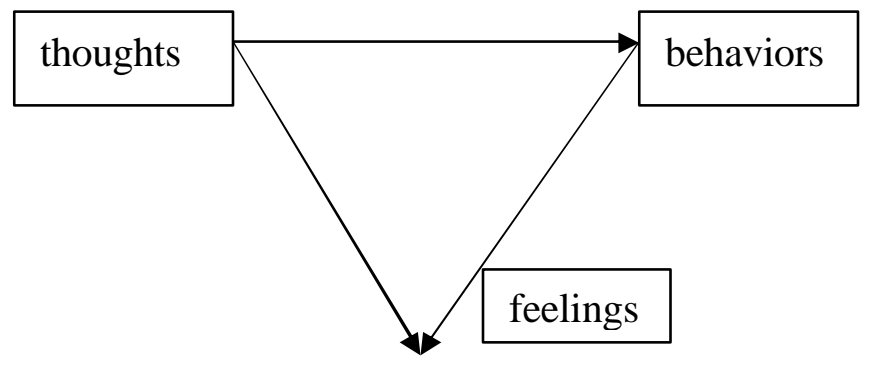

An example: Adopted mom won't let child have candy before dinner.

Child's thoughts: "If she really was my mom, I could have what I want!"

> New thought: "Most moms won't let their kids have candy before dinner"

Child's feelings: mad and sad

$>$ New feelings: calm, reassured.

Child's behavior: yells at mom "you are not my mom!"

> New behavior: child no longer yells at mom when she can’t have candy before dinner.

Exploration of negative behaviors is also done using the PACE stance through the intersubjective dialogue to gain insight into what may prompt negative behaviors. The child is then encouraged to choose coping skills they might use in the future that could prevent the negative behavior.

At the next appointment, psycho-education with the parents/caregivers and child is done separately. Again the child has an opportunity to continue playing the Journey to the Wild Divine Game while the therapist is meeting with the parents/caregivers. The parent/caregiver is given general information regarding physical, emotional, or sexual abuse, or domestic violence, or foster care and/or adoption, or traumatic grief (whatever is most appropriate for the child). This may take anywhere between 1-3 sessions depending on the developmental age of the child. The child is given age appropriate generalized educational information on physical, emotional, sexual abuse or domestic violence, or on foster care, adoption or traumatic grief (whichever is most appropriate). The child's feelings are closely monitored for any reactivity. The parents/caregivers are encouraged to also monitor the child closely as well and to maintain attunement with their child. The child and parents/caregiver are then brought together in a joint session and a quiz game such as What Do You Know? (Deblinger, Neubauer, Runyon \& Baker, 2006) is played and score is kept. The child generally leaves the session feeling like an expert in the subject that was discussed, which helps to build their self esteem, and trust in the therapist and parents/caregivers. 
Generally at the next session, again meeting separately with the parents/caregivers and child, psycho-education is reviewed and any questions are answered. The child's behaviors are discussed utilizing the DDP model. Also at this session gradual exposure of the child's traumatic experience is introduced utilizing TF-CBT and/or EMDR (Eye Movement Desensitization and Reprocessing) (Shapiro, 2001) and the trauma narrative or life story is started.

EMDR is utilized when a child is uncomfortable with discussing the traumatic memories or is struggling with the affect associated with the traumatic event. EMDR is another evidence-based practice model(Tinker \& Wilson, 1999). In this therapy model distressing memories related to the trauma are identified, desensitized and reprocessed so that the child can have a more coherent narrative (a logical or orderly way in which to organize and create meaning from the traumatic events). In addition any triggers (reminders of the traumatic event) that my cause hyper arousal (a state of responsiveness to sensory stimulation or excitability) or behavioral problems are identified and reprocessed. A positive template (positive thought or memory) for future relating is also developed (Shapiro, Kaslow, Maxfield, 2007). For example if a child perceives the adults in her life as abusive and uncaring, a new image or thought is developed regarding the new parents/caregivers. The child is asked to talk about a time when the new parents/caregivers were kind, comforting and caring toward her. This thought is then processed using the bilateral eye movements or taps. The eye movements or tapping on palms used in EMDR is done in a systematic way. It involves bilateral stimulation (alternating right and left) while the child thinks of the traumatic event (the memory of the event), thoughts about his or her self, and the feelings associated with the event. EMDR is believed to impact two different types of networks, which facilitates reprocessing of the information about the traumatic event. First, EMDR seems to stimulate the memory network where the trauma is stored and then activates the information networks that can assist the individual in making sense out of the incident. When both networks operate simultaneously during the eye movement sets or tapping, it appears that the traumatic information is rapidly processed and traumatic reactions such as fear, panic, despair, and grief are replaced by more positive and helpful thoughts (Shapiro, 2007). When the trauma appears to be an isolated incident, the traumatic symptom can be cleared within one or two sessions. But when multiple traumatic events are involved then the time to heal may be longer (Lovett, 1999). As the child begins to have more success behaviorally, EMDR is used to strengthen related positive feelings and sensations and negative cognitions are resolved. An example of a negative cognition a child might have when they witnessed domestic violence would be "I should have done something". Resolution of this negative cognition might be "I did the best I could" or "I am safe now". This therapy model requires several hours of specialized training for individuals with a Master's degree or higher.

The creation of the trauma narrative then begins using either the TF-CBT model or EMDR model depending on the child's needs. The trauma narrative is also referred to as gradual exposure. The child is encouraged to talk about the traumatic event, and describe his or her feelings and thoughts about the event as the therapist writes or types the information. The goal is to separate thoughts and/or reminders of the traumatic event from the overwhelming negative feelings, such as extreme fear, helplessness, and shame or rage (Cohen, Mannarino \& Deblinger, 2006). Creating the entire trauma narrative may take several sessions. It is recommended that the creation of the trauma narrative be done with the child and therapist and later without the child present reviewed with the parents. This is done in order to minimize the dysregulation of the parents and child. Once the child has created their trauma narrative, the negative cognitions are identified, explored and corrected thereby re-creating a more coherent narrative which contains more accurate and helpful thoughts (e.g. negative cognition regarding child sexual abuse: 'It's my fault, I should never have worn that sundress" > helpful thought: "It doesn't matter what I wear, he should never have touched me..."). For children who experience multiple foster placements and/or multiple traumatic events, it is suggested that they create a "life narrative" rather than a trauma narrative (Cohen, Mannarino, Deblinger, 2006). A "life narrative" would include details of their life experiences thereby gaining mastery over horrifying aspects of the traumatic events by talking about their thoughts and feelings regarding the traumatic events. Once mastery/control is achieved the symptoms that were 
associated with the trauma then decrease and behavioral issues are minimized (e.g. the child has recurring night time bed wetting, but once the child gains mastery over the traumatic events the bed wetting resolves).

The trauma narrative is reviewed with the parents/caregivers separate from the child during each consecutive session. There are times however that the child needs to have the parents/caregivers with them during the creation of the trauma narrative. Often this is true with children who have been adopted. This also allows the child an opportunit y to develop a more trusting and closer relationship with the parents/caregivers. Again this decision is based on the child's needs for feeling safe and secure. The parents/caregivers are prepared to help the child cope with feelings by responding to the child in a supportive manner, and they will encourage the child to talk to them about any problems that arise in the future. It is very important for the child to learn that the parent can tolerate discussing the trauma (Cohen, Mannarion, Deblinger, 2006).

The parents/caregivers are also expected to praise the child for the hard work they have accomplished. When the trauma narrative is complete the child shares the narrative with the parents/caregivers. Working through traumatic events can be very painful, but the outcome provides the child and family with an opportunity to connect on a deeper level than ever before and a new more coherent narrative brings understanding and resolves the shame the child may have felt regarding the traumatic event. The child is able to let go of the negative feelings/shame (fear, frustration or anger) they had about themselves as well as the negative thoughts ("I should have done something"). The child is then able to create new thoughts ("I did the best I could") and develop more positive feelings (confident, relaxed or calm).

The next step in TF-CBT is to teach the child about personal safety. This teaches the child personal boundaries that the body is private, communication of feelings and desires, paying attention to "gut" feelings, identifying people and places that provide safety, learning the difference between secrets and surprises, and asking for help until help is provided (Cohen, Mannarino, Deblinger, 2006). Parents are expected to practice these skills with the child at home to ensure that the child understands and can identify possible dangers in their environment. If using the EMDR model at this point, the child would develop a future template (thoughts or ideas) in which to feel safe. For example the child would develop an appropriate response for being touched by an older child or adult on his/her private parts, then the child would imagine the response during bilateral eye movements or taps, the child would then say what they saw his/herself doing (yelling leave me alone and running away and getting help from an adult they trust) and how he/she felt ("brave") during this exercise. In this step when utilizing the DDP model the parents/caregivers would continue to provide an accepting environment where safety remains key. This is accomplished through open communication so that the child can feel free to express feelings and thoughts about their experiences in the future. It may take 8-12 sessions to resolve the traumatic experiences. There are times when the therapy may be extended beyond 12 sessions in order to deepen the closeness that the child has begun to develop with the parents/caregivers and to further assist those children who have experienced multiple traumas.

For many of these children they feel less exposed, and less vulnerable following resolution of the traumatic experiences. They seem to feel stronger after telling their narrative. They don't have to keep their feelings or thoughts secret any more. They then begin to form a deeper attachment and a more trusting relationship with their parents/caregivers. The intersubjective dialogue then continues between the child and parents/caregiver with little prompting from the therapist. The family is then ready to terminate therapy.

For some of these children they are able to move on with their life with their families but for others they may return periodically for a "booster" session. A "booster" session might be needed when a new developmental milestone is faced, such as transitioning to middle school, high school or dating, or 
when sensory processing issues reoccur, or when new social skills need to be taught or reviewed, or if a significant loss has occurred. For others the integration of the coherent narrative maybe a life long process. As the child grows and matures into adulthood the narrative may need to be reviewed and reworked to gain clarity and insight into their current life experiences as an adult.

Multiple treatment approaches are often necessary to meet the unique needs of these very special children and their families. Once the trauma symptoms have resolved then the child and family are able to integrate the coherent narrative into their life together. The child is then able to build a level of responsiveness associated with emotional accessibility and emotional engagement that is seen in a secure attachment. The child's core self is then strengthened and normal growth of closeness, empathy, understanding and healthy dependency is restored.

\section{References}

Amaya-Jackson, L. \& DeRosa, R. (2007). Treatment considerations for Clinicians in Applying EvidenceBased Practice to Complex Presentations in Child Trauma. Journal of Traumatic Stress, 20, 379390.

American Psychiatric Association (APA), (2000). Diagnostic and statistical manual of mental disorders (Fourth edition-Text revision. Washington, DC: Author.

Bell, C. (2003). The Journey to the Wild Divine. Colorado: The Wild Divine Project.

Childswork/Childsplay, LLC. (1996). The Coping Skills Game, New York: A Guidance Channel Company.

Cline, F., Fay, J. (1990). Parenting with Love and Logic. Colorado: Pinon Press.

Cohen, J., Mannarino, \& A., Deblinger, E. (2006). Treating Trauma and Traumatic Grief in Chidren and Adolescents. New York: Guilford Press.

Davies, D., (2004). Child Development $2^{\text {nd }}$ Ed. New York: Guilford Press.

Deblinger, E.,Neubauer, F., Runyon, M., \& Baker, D., (2006). What do You Know? New Jersey: @NJ Cares Institute; funded by SAMHSA \& NCTSI.

Hage, D., (2003). Therapeutic Parenting. Colorado: Families by Design Publications.

Hughes, D., (2006). Building the Bonds of Attachment $2^{\text {nd }}$ Ed. Maryland: Jason Aronson

Hughes, D., (2007). Attachment-Focused Family Therapy. New York: W. W. Norton \& Company.

James, B., (1989). Treating Traumatized Children. New York: Free Press.

Levy, T., (Ed.) (2000). Handbook of Attachment Interventions. San Diego: Academic Press

Lovett, J. (1999). Small Wonders, Healing Childhood Trauma with EMDR. New York: Free Press.

McCammon,S., Spence, S., \& Friensen, B., (2001). Promoting Family Empowerment Through Multiple Roles. Journal of Family Social Work, 5, 1-24. 
Moran, M. (2007). Developmental Trauma Merits DSM Diagnosis, Experts Say. Psychiatric News, 42, 20.

National Clearinghouse on Child Abuse and Neglect Information, (2001). In Focus: Understanding the Effects of Maltreatment on Early Brain Development. National Clearinghouse on Child Abuse and Neglect Information, U.S. Department of Health and Human Services. http://nccanch.acf.hhs.gov

Pacifici, C., Chamberlain, P., \& White, L. (2002). Off Road Parenting. Oregon: Northwest Media, Inc.

Scaer, R., (2005). The Trauma Spectrum. New York: W. W. Norton \& Company.

Shapiro, F., (2001). Eye Movement Desensitization and Reprocessing $2^{\text {nd }}$ Ed. New York: Guilford Press.

Shapiro, F., Kaslow, F., \& Maxfield, L. (Eds.). (2007). Handbook of EMDR and Family Therapy Processes. New Jersey: John Wiley \& Sons, Inc.

Solomon, M., \& Siegel, D. (Eds.). (2003). Healing Trauma. New York: W. W. Norton \& Company.

Thomas, N., (1997). When Love is Not Enough. Colorado: Families by Design Publications.

Tinker, R., \& Wilson, S., (1999). Through the Eyes of a Child, EMDR with Children. New York: W. W. Norton \& Company.

van der Kolk, B., McFarlane, A., \& Weisaeth, L. (Eds.) (1996). Traumatic Stress. New York: Guilford Press.

\section{Author Contact Information:}

Dianna Aideuis, RN, MSW, LCSW

Private Practice

New Hope Counseling Services, P.A.

500-C Dexter St.

Greenville, NC 27834

Phone: 252-355-3900

Fax: 252-355-3995

E-mail: reframing@earthlink.net 